\title{
Establishment of a colorectal cancer nude mouse visualization model of HIF-1 $\alpha$ overexpression
}

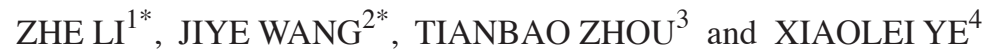 \\ ${ }^{1}$ Department of Thyroid and Breast Surgery, The Shanghai Tenth People's Hospital, Tong Ji University, Shanghai 200072; \\ ${ }^{2}$ Department of Criminal Science and Technology, Zhejiang Police College, Hangzhou, Zhejiang 310053; \\ ${ }^{3}$ Hepatobiliary Surgery Centre, The Ningbo No. 2 Hospital, Ningbo, Zhejiang 315010; \\ ${ }^{4}$ Division of Drugs and Pharamcology, Ningbo Institute of Medical Sciences, Ningbo, Zhejiang 315020, P.R. China
}

Received October 14, 2014; Accepted November 26, 2015

DOI: $10.3892 / \mathrm{ol} .2016 .4287$

\begin{abstract}
The aim of the present study was to establish a model of tumor cell growth and visualize HIF-1 $\alpha$ overexpression in a nude mouse xenograft model of colorectal cancer (CRC). In the study, HIF-1 $\alpha$ lentiviral vector and helper plasmid were co-transfected into 293T packaging cells using a liposome method, and the virus was collected following transfection and used to infect CRC SW480, SW620, LoVo and HCT116 cells. Puromycin was used for the selection and large-scale amplification of the stable HIF-1 $\alpha$ expression of green fluorescent protein (GFP)-positive cells. HIF-1 $\alpha$-expressing cells were injected intraperitoneally into a nude mouse xenograft model, and resulting tumor nodules was separated and confirmed using an inverted fluorescence microscope. The results demonstrated that HIF-1 $\alpha$ was not expressed in CRC cells in normoxic conditions. When treated with $\mathrm{CoCl}_{2}$, the expression of HIF-1 $\alpha$ could be induced in all the cancer cell lines, except SW480. HIF-1 $\alpha$ was highly expressed following infection with lentiviral particles. Stable expression of HIF-1 $\alpha$ promoted migration in the SW480 cells. Following intraperitoneal injection of nude mice with SW480-HIF-1 $\alpha$, a significant number of tumor nodules formed in the intestinal wall compared with the controls $(\mathrm{P}<0.05)$. The successful construction of the dual expression HIF-1 $\alpha$ and GFP visualization xenograft model provides a good foundation for the screening of HIF-1 $\alpha$-related functions and for investigating the therapeutic potential of drugs that target HIF-1 $\alpha$.
\end{abstract}

Correspondence to: Mr. Zhe Li, Department of Thyroid and Breast Surgery, The Shanghai Tenth People's Hospital, Tong Ji University, 301 Middle Yangchang Road, Shanghai 200072, P.R. China E-mail: lizhe@medmail.com.cn

Mr. Xiaolei Ye, Division of Drugs and Pharmacology, Ningbo Institute of Medical Sciences, 247 Renmin Road, Ningbo, Zhejiang 315020, P.R. China

E-mail: yexiaolei@gmail.com

*Contributed equally

Key words: colorectal cancer, xenograft model, stable transfection, lenti virus, HIF-1 $\alpha$, green fluorescent protein

\section{Introduction}

Colorectal cancer (CRC) is a common gastrointestinal malignancy, and worldwide, it is ranked the third most frequent type of cancer in men and the second most frequent type in women. In North America, New Zealand, Western Europe, Japan and other economically developed areas, CRC ranks first or second in frequency among all cancers in men. The clinical presentation of CRC and the associated mortality has followed an increasing trend (1). Hypoxia is a common pathological feature of solid tumors, such as CRC, and is caused by low levels of tumor cell differentiation and rapid tumor growth with insufficient growth of blood vessels, resulting in an insufficient blood supply to the tumor (2).

Hypoxia induces the high expression of hypoxia-inducible factor- $1 \alpha$ (HIF-1 $\alpha$ ). A previous study identified that $\sim 55 \%$ of CRC patients exhibit elevated expression levels of HIF-1 $\alpha$ (3). Activated HIF-1 $\alpha$ binds to the hypoxia response element and forms the response element binding protein with other transcription factors, to regulate the expression of $\sim 60$ target genes. These factors include vascular endothelial growth factor (VEGF) and basic fibroblast growth factor, which are now recognized as the most important angiogenesis-promoting factors (4). HIF-1 $\alpha$ expression is important in the development of CRC, and transient HIF-1 $\alpha$ expression in colon cancer cells can rapidly increase VEGF expression (5). Furthermore, overexpression of HIF-1 $\alpha$ in CRC cells injected subcutaneously into nude mice can promote cell proliferation and angiogenesis (6). As hypoxia can induce the epithelial-mesenchymal transition of CRC cells, thus promoting metastasis (7), hypoxia-induced gene expression profiles may be used as independent prognostic markers for predicting the outcome of stage II and III colon cancers (8).

Hypoxia is important in promoting CRC occurrence, angiogenesis and metastasis. It is important to identify and verify the key gene that is responsible for regulation of tumor angiogenesis and metastasis following activation of HIF-1 $\alpha$. The present study aimed to investigate the practical significance of HIF-1 $\alpha$ targeting for the treatment and prevention of CRC angiogenesis, invasion and metastasis. A lentiviral vector containing green fluorescent protein (GFP) and HIF-1 $\alpha$ was constructed and used to infect CRC cell lines in vitro, for coexpression of these proteins in stably-transfected cell lines. Establishing a 
visual xenograft nude mouse model may lay the foundation for studying the biological functions of HIF-1 $\alpha$ in CRC in vivo.

\section{Materials and methods}

Materials. The lentiviral vectors pLV-TRC-EGFP, $\mathrm{p} \Delta 8.91$ (virus helper plasmid) and pMD2.G (helper plasmid, encoding VSV-G), and 293 T cells were purchased from Beijing Zhongshan Golden Bridge Biotechnology Co., Ltd. (Beijing, China). The human CRC SW480, SW620, LoVo and HCT116 cell lines were purchased from the cell bank of the Shanghai Institute of Biochemistry and Cell Biology (Shanghai, China). Tissue culture flasks and 96-well plates were purchased from Greiner Bio-One GmbH (Frickenhausen, Germany). Minimum essential medium (MEM) and Dulbecco's modified Eagle's medium (DMEM) were purchased from Life Technologies (Grand Island, NY, USA), and $\mathrm{X}$-tremeGENE transfection reagent was purchased from Roche Diagnostics (Basel, Switzerland). The plasmid miniprep kit and molecular cloning enzymes were purchased from Sangon Biotech Co., Ltd. (Shanghai, China). NheI was obtained from New England BioLabs, Inc. (Ipswich, MA, USA) and agarose gel was purchased from Biowest (Nuaillé, France). High-fidelity Taq polymerase was purchased from Toyobo Co., Ltd., (Osaka, Japan), LB medium was obtained from Caisson Laboratories (Logan, UT, USA) and puromycin, polybrene and ampicillin were purchased from Sigma-Aldrich (St. Louis, MO, USA). Antibodies used for western blotting were purchased from Santa Cruz Biotechnology, Inc., (Dallas, TX, USA) and the nude mice was purchased from Changzhou Card Vince Laboratory Animal Co., Ltd. (Luoyang, China).

Construction of overexpressing lentiviral vector. i) Primers targeting the HIF-1 $\alpha$ open reading frame (ORF) of the pBabe vector (obtained from Professor Ji Hongbin, Laboratory of the Shanghai Institute of Biochemistry and Cell Biology, Shanghai, China) were designed with an NheI restriction site at the $5^{\prime}$ end. Primers were designed using Vector $\mathrm{NTI}^{\circledR}$ software version 10.0 (Thermo Fisher Scientific, Inc., Waltham, MA, USA), which covered the open reading frame of HIFl $\alpha$. High-fidelity Taq polymerase was used for polymerase chain reactions (PCR) to amplify the ORF DNA fragment, and the DNA template was removed using DpnI. Following digestion with NheI, the purified PCR product was ligated to the pLV-TRC-EGFP plasmid. ii) $\mathrm{DH} 5 \alpha$ competent cells: $2 \mu \mathrm{l}$ of the ligation products were transformed into $25 \mu \mathrm{l}$ Escherichia coli cells $\left(\sim 5 \times 10^{7}\right.$ cell $\left./ \mathrm{ml}\right)$ with DH5 $\alpha$ that had been plated on a petri dish containing $100 \mathrm{~g} /$ $\mathrm{ml}$ ampicillin in lysogeny broth (LB) medium and incubated at $37^{\circ} \mathrm{C}$ overnight. Three monoclonal colonies were picked from each dish and inoculated into $3 \mathrm{ml} \mathrm{LB}$ medium with ampicillin, and then incubated overnight at $37^{\circ} \mathrm{C}$ on a shaker. iii) Identification of the recombinant plasmid: The plasmid was extracted using a miniprep kit (Qiagen GmbH, Hilden, Germany), and identified by digestion with NheI. The plasmid was identified using $1 \%$ agarose gel (Biowest, Nuaillé, France) electrophoresis with electrophoresis apparatus (HE120 Multifunctional Horizontal Gel Electrophoresis Cell; Tanon Science \& Technology Co., Ltd., Shanghai, China), and the present study refers to this plasmid as pLV-HIF1 $\alpha$-EGFP. iv) Sequencing of expression vectors: Digestion using the appropriate restriction enzymes confirmed the correct fragments were isolated and amplified from bacterial cultures, and these were sent for sequencing (Biosune Biotechnology Co., Ltd., Shanghai, China). v) Large-scale plasmid extraction: The sequenced pLV-HIF $1 \alpha$-EGFP plasmid was amplified in LB broth using a Plasmid kit (Qiagen, Inc., Valencia, CA, USA) for large-scale plasmid extraction, and the isolated plasmids were subjected to quality control. Isolated plasmids were examined using a NanoDrop $2000^{\mathrm{TM}}$ spectrophotometer (Thermo Fisher Scientific, Inc.), and an optical density value of 1.8 (at 260/280 nm) was treated as the quality control.

Lentiviral particles packaging and titer testing. The 293T cells were plated onto tissue culture the day prior to transfection. On the day of transfection, cell confluency did not exceed $80 \%$. The $\mathrm{X}$-tremeGENE reagent was used to transfect the expression plasmid containing pLV-HIF1 $\alpha$-EGFP and the two packaging plasmids p $\Delta 8.91$ and pMD2.G into the 293T cells, according to a mass ratio of 10:10:1. Following $12 \mathrm{~h}$ of incubation at $37^{\circ} \mathrm{C}$, the complete medium was replaced, and after $24 \mathrm{~h}$ of incubation, expression of GFP was observed in the $293 \mathrm{~T}$ cells, using a fluorescence microscope (Axio Imager 2; Zeiss, Oberkochen, Germany). Cell culture supernatants were collected during 48-72 h, and virus particles were passed through a $0.45-\mu \mathrm{m}$ filter and stored at $4{ }^{\circ} \mathrm{C}$. The purified virus particles were titred as follows: $293 \mathrm{~T}$ cells in the logarithmic growth phase were plated onto 96 -well plates $\left(8,000\right.$ cells/well) and cultured at $37^{\circ} \mathrm{C}$ in DMEM containing 2\% fetal bovine serum (FBS; Biological Industries, Beit-Haemek, Israel) until 30-50\% confluency was reached, after $16 \mathrm{~h}$. The purified virus suspension was diluted 1:10 with DMEM containing 2\% FBS, and used to replace the medium in the 96-well plates. Each virus dilution was used to infect two wells. Following incubation overnight at $37^{\circ} \mathrm{C}$, the virus-containing medium was discarded and replaced with DMEM containing 10\% FBS. At day 5, the number of fluorescent cells in each virus dilution was observed under a fluorescence microscope. Transducing units (TU) were calculated as follows: Titer $=$ cells with fluorescence $\mathrm{x}$ dilution ratio, and the final lentivirus titer was $5 \times 10^{7} \mathrm{TU} / \mathrm{ml}$.

Lentiviral infection. CRC cells in the logarithmic growth phase were seeded into 6 -well plates. Following $12 \mathrm{~h}$ of culture, the supernatant was discarded and $200 \mu \mathrm{l} /$ well of virus suspension was added to medium containing polybrene (concentration is $4 \mu \mathrm{g} / \mathrm{m} 1$ ). At $8 \mathrm{~h}$ post-viral infection, the medium was changed to DMEM with $2 \%$ FBS. Following $48 \mathrm{~h}$ of cell culture, puromycin $(0.5 \mu \mathrm{g} / \mathrm{ml})$ was added to the medium. The culture medium was replaced every 2 days to remove dead cells. After screening with $0.5 \mu \mathrm{g} / \mathrm{ml}$ puromycin three times to determine which cells had undergone successful transfection, DMEM containing $10 \%$ FBS was added for $24 \mathrm{~h}$ and the cells were then collected in culture flasks for subsequent experiments.

Western blot analysis. The cells were rinsed twice with cold phosphate-buffered saline (PBS) and then lysed on ice in radioimmunoprecipitation assay lysis buffer. Following this, the cells were collected and the proteins were separated on a $10 \%$ acrylamide gel using sodium dodecyl sulfate-polyacrylamide gel electrophoresis and a Bio-Rad mini system (Bio-Rad Laboratories, Inc., Hercules, CA, USA). Separated proteins were then transferred to a polyvinylidene difluoride membrane (EMD 
Millipore, Billerica, MA, USA). Non-specific antibody binding sites were blocked for $1 \mathrm{~h}$ with 5\% skimmed milk (Nestlé, Vevey, Switzerland). Anti-HIF-1 $\alpha$ mouse monoclonal anti-human (cat. no. ab82832; dilution, 1:1,000; Abcam, Cambridge, MA, USA), anti-VEGF rabbit polyclonal anti-human (cat. no. ab46154; dilution, 1:1,000; Abcam) and anti-pyruvate kinase isozyme M1 (PKM1) rabbit polyclonal anti-human (cat. no. ab156849; dilution, 1:1,000; Abcam) primary antibodies were incubated with the membrane at $4^{\circ} \mathrm{C}$ overnight. Subsequent to being washed with Tris-buffered saline plus Tween 20 (TBST), the horseradish peroxidase-conjugated rabbit anti-mouse (cat. no. 7076; dilution, 1:3,000; Cell Signaling Technology, Inc., Danvers, MA, USA) and goat anti-rabbit (cat. no. 7074; dilution, 1:3,000; Cell Signaling Technology) IgG secondary antibodies were added at room temperature for $1 \mathrm{~h}$. The membrane was then washed three times with TBST. Electrochemiluminescence reagent was then applied to the membrane (Life Technologies), and film was developed using a darkroom.

Cell migration assay. Matrigel (stored at $-80^{\circ} \mathrm{C}$ ) was allowed to melt at room temperature, and was then diluted 1:4 with MEM. A total of $50 \mu \mathrm{l}$ of the solution was added to each Transwell chamber (Corning Inc., Corning, NY, USA) and allowed to dry at $4^{\circ} \mathrm{C}$. The excess solution was absorbed and $50 \mu 1$ of medium was added to each chamber and incubated at $37^{\circ} \mathrm{C}$ for $30 \mathrm{~min}$. The cells were suspended at a density of $5 \times 10^{5}$ cells/ $\mathrm{ml}$ in MEM. Cell suspension (100 $\mu \mathrm{l})$ was placed in each upper chamber, and $600 \mu \mathrm{l}$ MEM containing 10\% FBS was placed into the lower compartment. Following incubation for 3-6 h, the cell culture medium of the upper and lower chambers was discarded and the membrane was removed and dried. Crystal violet solution (600 $\mu \mathrm{l}$; diluted 1:4 in 2\% ethanol) was applied to the membranes for $30 \mathrm{~min}$. The membranes were washed twice with PBS, and the upper surface of each membrane was wiped with a cotton swab to remove non-migrated cells. The cells that had migrated through the membrane were visualized using a microscope (CX21BIM-SET5; Olympus Corp., Tokyo, Japan).

Establishment of an abdominal tumor nude mouse model. Logarithmic growth phase CRC cells were digested with $0.25 \%$ trypsin and washed once with fresh medium. The cell pellet was collected by centrifugation at $1,000 \mathrm{x} \mathrm{g}$ for $3 \mathrm{~min}$ and the supernatant was discarded. The cells were washed twice with sterile saline and counted. The concentration of live cells was adjusted to $1 \times 10^{7} / \mathrm{ml}$. Prior to inoculation, the skin of the nude mice was disinfected with alcohol. The cell suspension $(200 \mu \mathrm{l})$ was injected intraperitoneally into mice with a $1-\mathrm{ml}$ syringe, to confer a cell mass of $2 \times 10^{6}$ cells/mouse. After 14 days, the mice were dissected to remove any nodules and to confirm the expression of GFP under a fluorescence microscope.

Statistical analysis. All data are expressed as the mean \pm standard deviation. The paired Student's t-test and $\chi^{2}$ test were used to determine statistical significance. Statistical analysis was performed with GraphPad Prism software version 5 (GraphPad Software, Inc., La Jolla, CA, USA). $\mathrm{P}<0.05$ was considered to indicate a statistically significant difference.

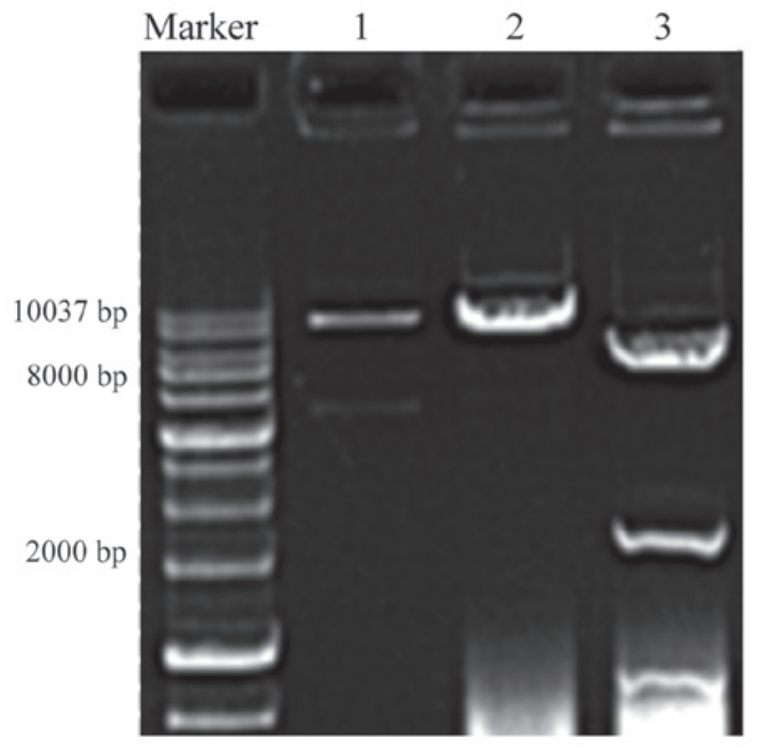

Figure 1. Plasmids analyzed by enzymatic digestion. Lane 1 contains pLV-HIF1 $\alpha$-GFP, lane 2 contains linearized plasmid, and lane 3 contains the plasmids following digestion by NheI. HIF-1 $\alpha$, hypoxia-inducible factor-1 $\alpha$; GFP, green fluorescent protein.

A

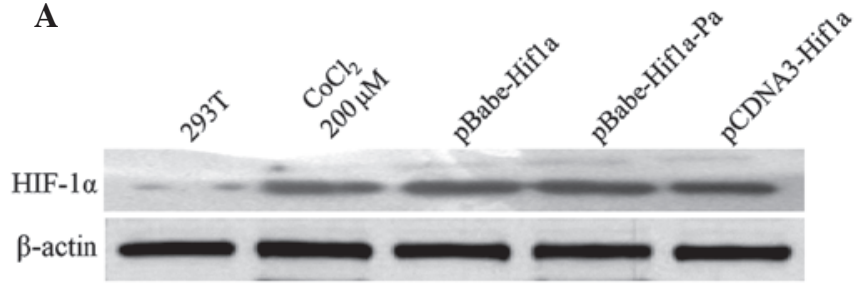

B
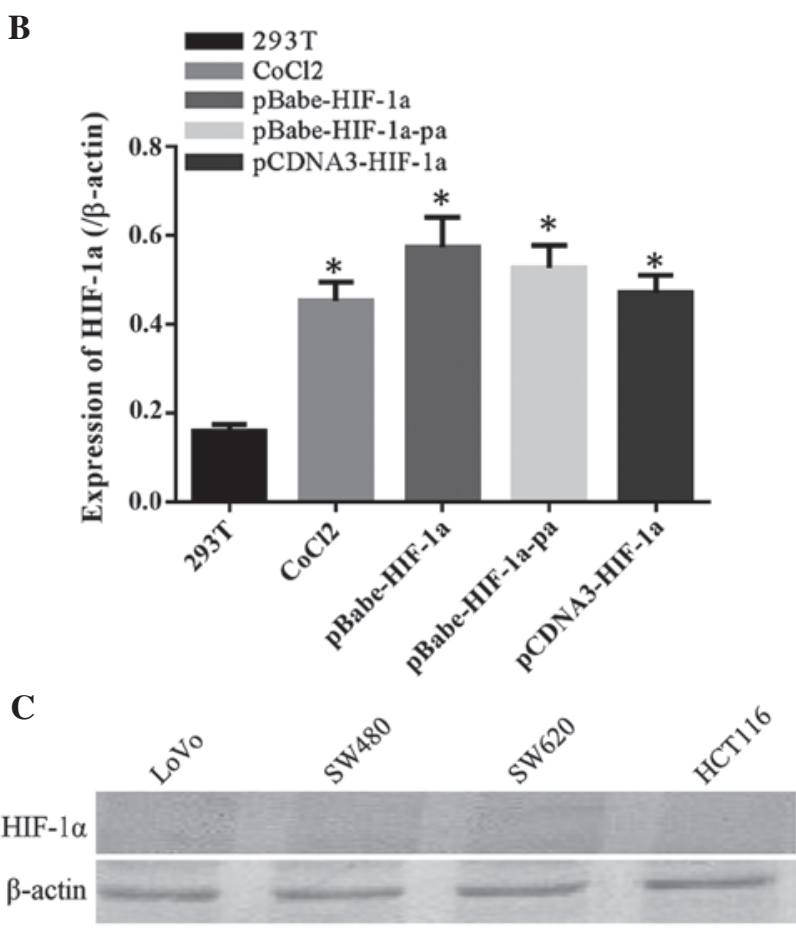

Figure 2. HIF-1 $\alpha$ protein is not expressed in LoVo, SW480, SW620 and HCT116 colorectal cancer cell lines under normal conditions, and is not detectable by western blotting. (A) HIF-1 $\alpha$ protein expression was detected and (B) quantified in $293 \mathrm{~T}$ cells following treatment for $48 \mathrm{~h}$ with $200 \mu \mathrm{M}$ $\mathrm{CoCl}_{2}$, pBabe-HIF-1 $\alpha$, pBabe-HIF- $1 \alpha$-pa and pCDNA3-HIF-1 $\alpha$ plasmids. (C) Western blot analysis of HIF-1 $\alpha$ protein expression levels in various colon cancer cell lines. ${ }^{*} \mathrm{P}<0.05$ vs. untransfected $293 \mathrm{~T}$ cells. HIF-1 $\alpha$, hypoxia-inducible factor- $1 \alpha$. 

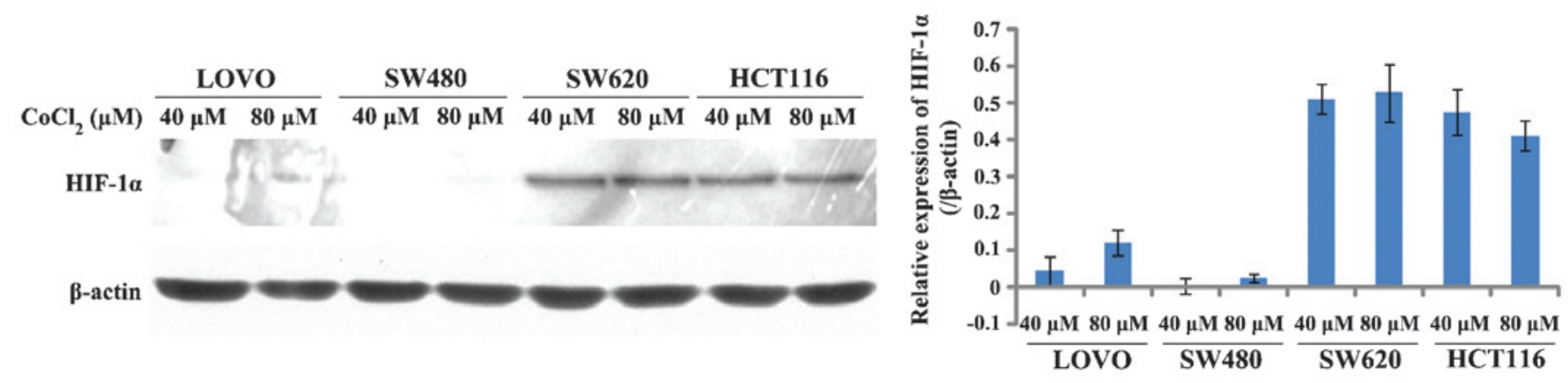

Figure 3. Expression of HIF-1 $\alpha$ in colorectal cancer cells following treatment with $\mathrm{CoCl}_{2}$ for $24 \mathrm{~h}$. LoVo, SW480, SW620 and HCT116 cell lines were treated with 40 or $80 \mu \mathrm{M} \mathrm{CoCl}_{2}$ for $24 \mathrm{~h}$, and the protein level of HIF-1 $\alpha$ was detected by western blotting. HIF-1 $\alpha$, hypoxia-inducible factor-1 $\alpha$.

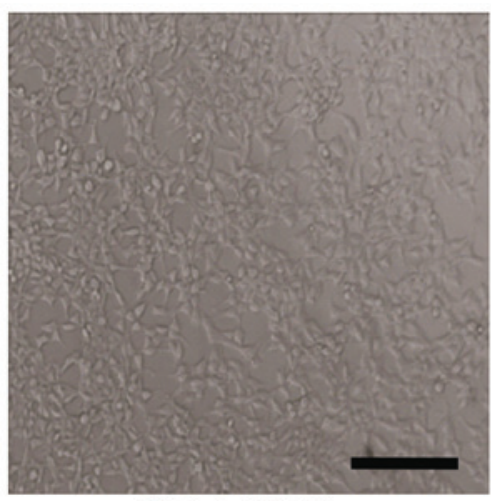

Bright field

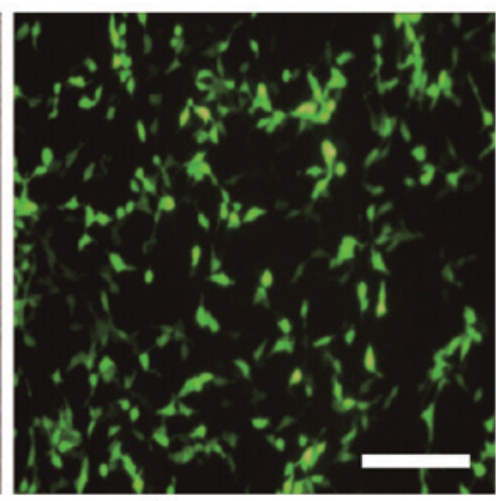

Fluorescence

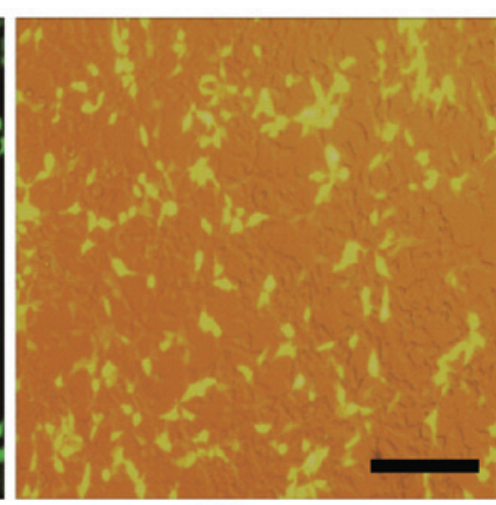

Merge

Figure 4. Microscopy of 293T cells transfected by PLV-HIF1 $\alpha$-GFP. The centre panel demonstrates expression of the GFP reporter. GFP, green fluorescent protein; HIF-1 $\alpha$, hypoxia-inducible factor-1 $\alpha$.

\section{Results}

HIF-1 1 vector construction, identification and overexpression. The pLV lentiviral vector was used to stabilize expression of GFP and HIF- $1 \alpha$ by molecular cloning. Molecular analysis of the plasmid identified two (8.8-kbp and 2.5-kbp) bands, which were cut out following incubation with the NheI restriction enzyme. The correctly identified plasmid was sent to Biosune Biotechnology Co., Ltd. for nucleotide sequencing using universal primers, and this confirmed that the correct nucleotide sequence was present. Thus, the constructed plasmid could be used for the transfection of the 293T cells and the expression of the coding sequence of HIF-1 $\alpha$ isoforms 1 and 3 (Fig. 1).

HIF-1 $\alpha$ expression levels in CRC. HIF-1 $\alpha$ expression was not detected under normal conditions in four different CRC cell lines, as shown in Fig. 2. Previous studies have indicated that $\mathrm{CoCl}_{2}$ can simulate cell hypoxia and induce the expression of HIF-1 $\alpha$ (9). Fig. 3 demonstrates that the SW620 and HCT116 cell lines had elevated levels of HIF-1 $\alpha$ following treatment with $\mathrm{CoCl}_{2}$ for $24 \mathrm{~h}$, while HIF-1 $\alpha$ expression in the LoVo cells was only slightly upregulated. HIF-1 $\alpha$ expression in the SW480 cells was not induced, because that SW480 was the low metastatic potential CRC cell lines, SW480 as a targeted infection cell lines for lentiviral infection after packaging could be better observed the effect of HIF-1 $\alpha$ overexpression on tumor biology.
HIF-1 $\alpha$ overexpression plasmid transfection and lentivirus packaging. The constructed pLV-HIF1 $\alpha$-GFP plasmid and the corresponding helper plasmid were used to transfect $293 \mathrm{~T}$ cells (Fig. 4). The transfection efficiency was $>80 \%$. The culture supernatant virus was collected for titer determination after transfected $48 \mathrm{~h}$ and the virus titer was $5-7 \times 10^{6}$ transduction units (TU)/ml. After the SW480 cells were infected with lentivirus for $72 \mathrm{~h}$, the expression of GFP was observed by fluorescence microscopy and cell morphology was analyzed, confirming effective lentivirus infection of the SW480 cells and expression of the target protein (Fig. 5). Next, puromycin was added, and after 3 weeks of screening, cell lines stably expressing HIF-1 $\alpha$ were obtained. Western blot analysis confirmed the screened stable SW480-HIF-1 $\alpha$ was successfully constructed (Fig. 6).

Stable HIF-1 $\alpha$ expression in SW480 cells following puromycin screening. Western blot analysis confirmed that HIF-1 $\alpha$ was highly expressed in the SW480 cells following puromycin screening (Fig. 6). In addition, the HIF-1 $\alpha$ downstream target genes, VEGF and PKM1, exhibited a certain degree of increased expression. VEGF exhibited the most significant upregulation, followed by PKM1, suggesting that the overexpression of HIF-1 $\alpha$ may have an extensive role in cells, inducing angiogenesis growth factors and promoting glycolysis.

Effects on cell migration after HIF-1 $\alpha$ stable expression. Transwell chamber mobility change was comparable prior to 

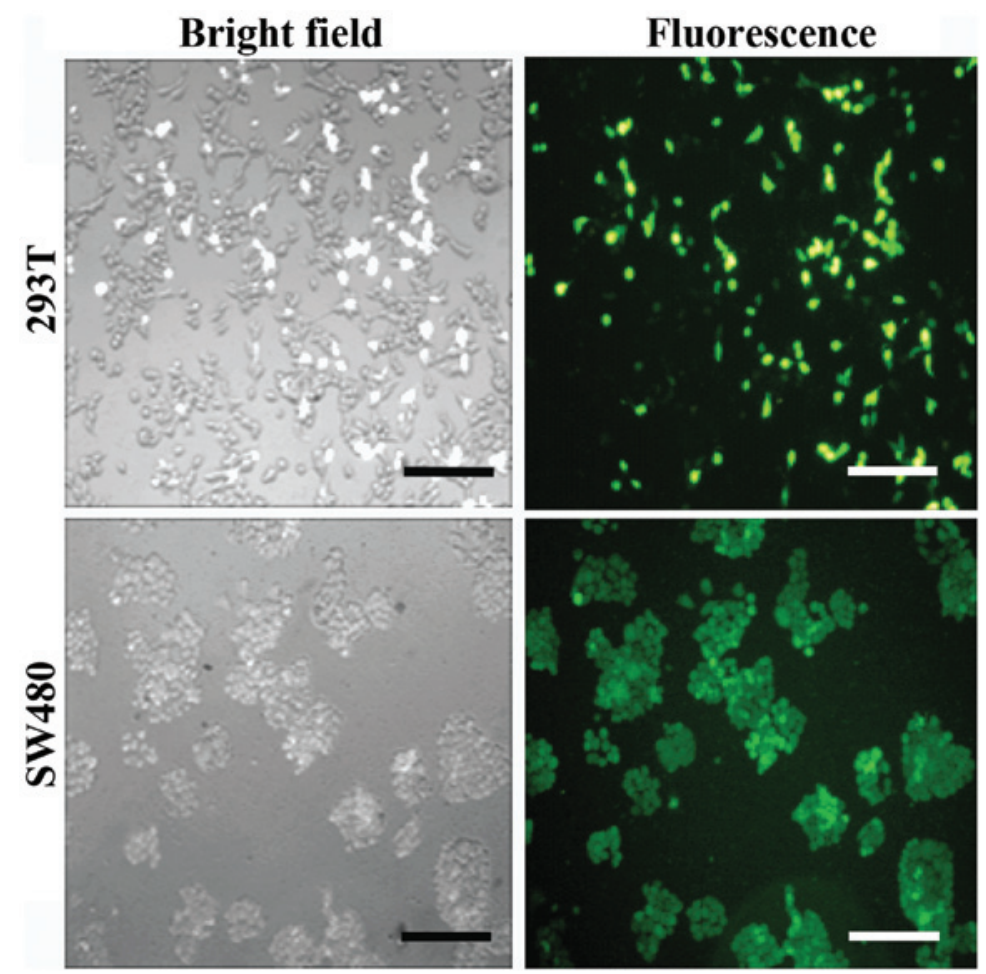

Figure 5. Cell morphology following infection with lentivirus particles. The left panels indicate bright field images of $293 \mathrm{~T}$ and SW480 cells (scale bar, $100 \mu \mathrm{m}$ ). Right panels indicate fluorescent microscopy of the green fluorescent protein-containing cells.
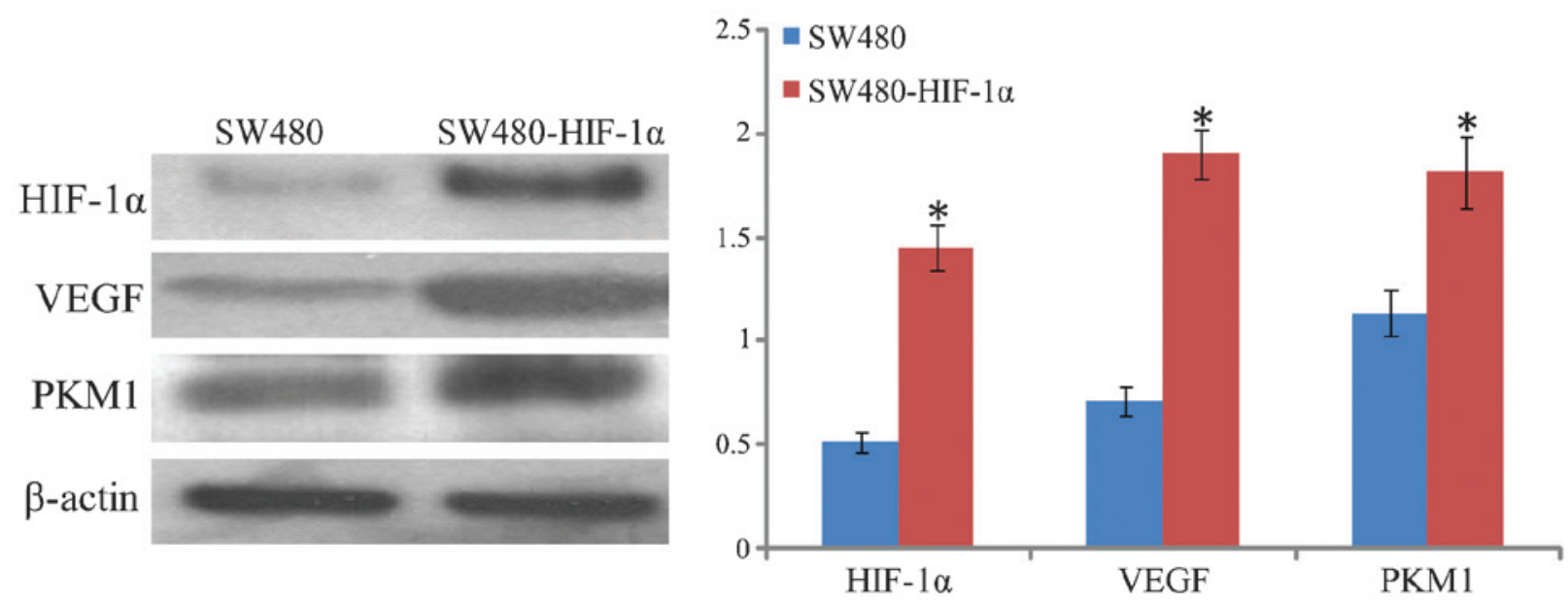

Figure 6. Stable expression of HIF-1 $\alpha$ following lentiviral infection. The protein levels of HIF-1 $\alpha$ and its downstream targets VEGF and PKM1 were detected in the SW480 and SW480-HIF-1 $\alpha$ cell lines. "P<0.05 compared with SW480. HIF-1 $\alpha$, hypoxia-inducible factor-1 $\alpha$; VEGF, vascular endothelial growth factor; PKM1, pyruvate kinase isozyme M1.

and following HIF-1 $\alpha$ overexpression; the results showed that prior to SW480-HIF-1 $\alpha$ stable overexpression, mobility had improved significantly $(\mathrm{P}<0.0001$; Fig. 7$)$, increased from $50 \pm 5$ cells/field to $250 \pm 11$ cells/field (x200 magnification). These data suggested that HIF- $1 \alpha$ overexpression significantly increased the invasion and migration of cells.

Construction of GFP/HIF-1 $\alpha$ dual expression xenografts in nude mice. CRC cells expressing GFP and HIF-1 $\alpha$ were injected into the abdomen of nude mice. After 14 days, nodules were dissected from the nude mice and counted (Fig. 8), Mice with SW480-HIF-1 $\alpha$ exhibited nude multiple nodules in the abdominal cavity (Fig. $8 \mathrm{~A}$ and B), with $15 \pm 4$ nodules/mouse compared with $4 \pm 1$ nodules/mouse in the control group; this difference was statistically significant $(\mathrm{P}<0.05)$. Subsequent to removal of the nodules, they were observed under a fluorescence microscope; nodules with green fluorescence expressed high levels of GFP and HIF-1 $\alpha$, present in the SW480 colon cancer cells (Fig. 8C).

\section{Discussion}

Gene expression in lentivirus-infected target cells is stable and persistent (10). Since the first use of the human immunodeficiency virus as a gene transfer vector, the ability has 


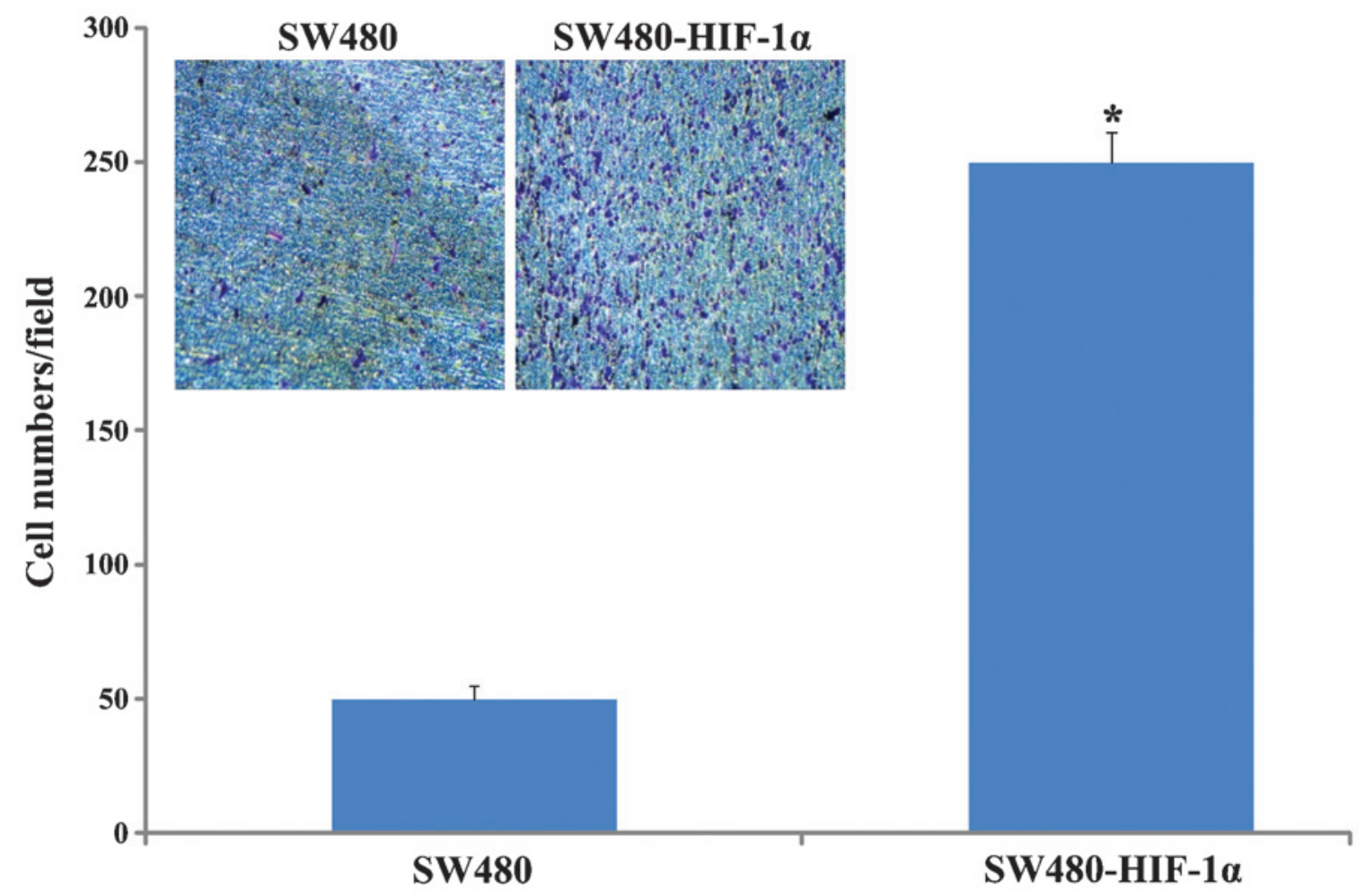

Figure 7. Cell migration activity of SW480 following HIF-1 $\alpha$ stable expression. Cell migration ability was compared using a Transwell assay with SW480 and SW480-HIF-1 $\alpha$ cells $(n=3)$. $P<0.05$ compared with SW480. HIF-1 $\alpha$, hypoxia-inducible factor-1 $\alpha$.
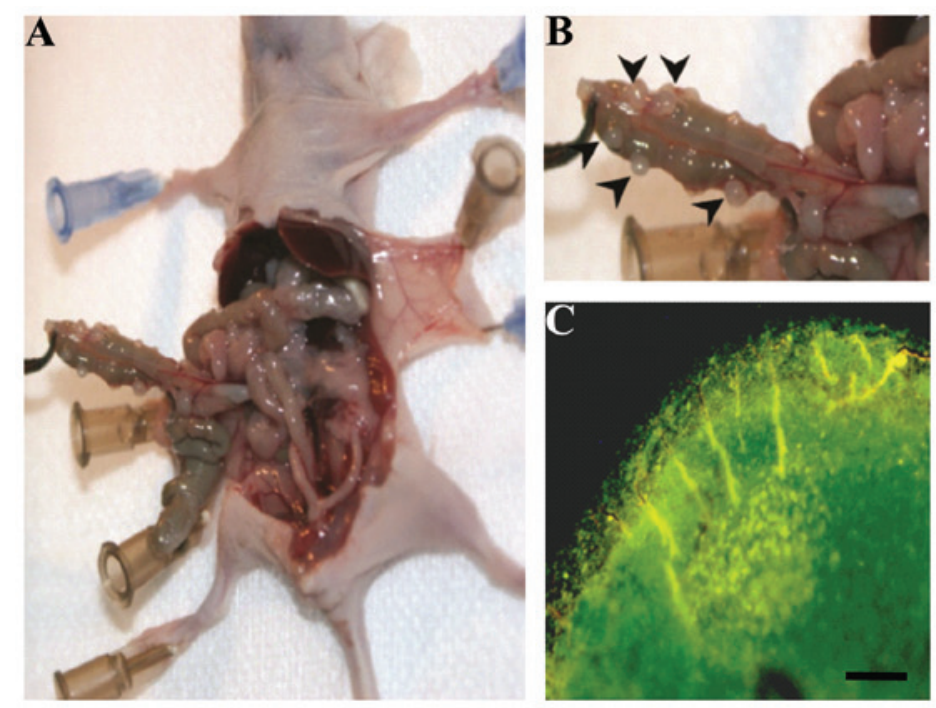

\section{D}

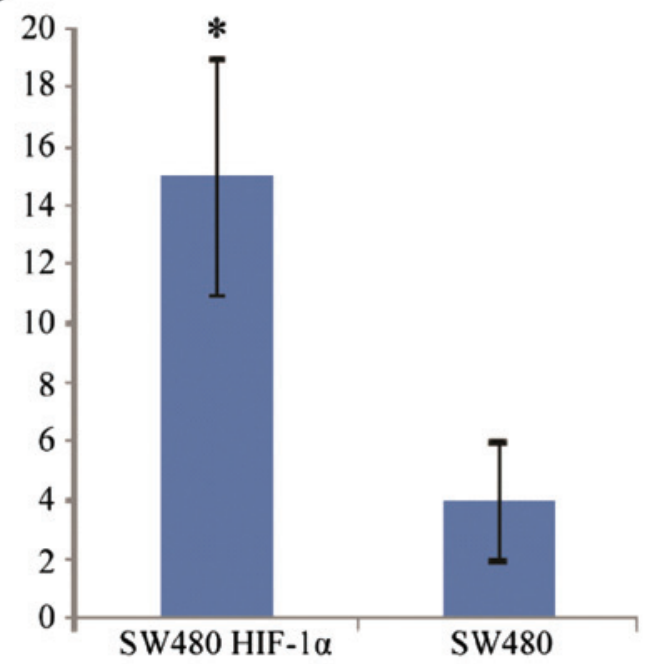

Figure 8. (A) Tumor nodules on the abdominal wall. (B) Representative images of tumor nodules on mouse colon, indicated by arrows. (C) Dissected nodules were exposed under a fluorescence microscope and images were captured (scale, $100 \mu \mathrm{m}$ ). (D) Number of tumor nodules. *P<0.05 compared with SW480. HIF-1 $\alpha$, hypoxia-inducible factor- $1 \alpha$.

existed to integrate a pseudovirus into the host genome. The expressed gene is not affected by cell division or the environment (11), therefore, the technique can significantly increase the expression levels of unstable proteins in cells (10). This technology constitutes a more efficient class of expression vectors, which not only aid basic cancer research, but can also be used for gene therapy in the clinic (12). HIF-1 $\alpha$ is an important factor in tumorigenesis and development, and there is extensive systematic research into its role in CRC. HIF-1 $\alpha$ is only expressed under hypoxic conditions, therefore, experimental study of its functions requires the use of hypoxic conditions or $\mathrm{CoCl}_{2}$, which simulates hypoxia in vitro. To generate hypoxic conditions for cell culture, researchers must use a humidified incubator with varying proportions of $\mathrm{O}_{2}$ and $\mathrm{CO}_{2}$ or a low oxygen chamber $\left(\mathrm{O}_{2}\right.$ may be downregulated to $3 \%$ using a multi-gas inhibitor) (13). These requirements add to the experimental cost and amount of apparatus required, as well as inducing a cellular stress response. In addition to the expression of HIF- $1 \alpha$, other proteins exist that are regulated by a stress response feedback. The use of high 
concentrations of $\mathrm{CoCl}_{2}$ may change the osmotic pressure in cells, and can exert other effects, such as inhibiting tumor cell proliferation (14). Thus, the traditional methods for inducing the expression of HIF-1 $\alpha$ are associated with a number of complicating factors that have made the elucidation of gene function more difficult (15); this may be the reason for the low availability of drugs currently targeting HIF-1 $\alpha$ (16).

HIF-1 $\alpha$ protein is abundantly expressed the majority of solid tumors, including colon, breast, pancrease, kidney, prostate, ovary, brain and bladder cancer $(6,17,18)$. In turn, inhibiting HIF-1 $\alpha$ may directly inhibit tumor formation (19), which is not conducive for studying the in vivo function of HIF-1 $\alpha$. Establishing GFP and HIF-1 $\alpha$ stable co-expression in CRC cell lines using lentiviral technology may solve the aforementioned problems. Furthermore, as HIF-1 $\alpha$ is hardly expressed in the normoxic state in tumor studies, it is vital to establish cell and animal models with with expression of HIF-1 $\alpha$ under normal conditions. The establishment of models in vivo and in vitro may improve our understanding of the role of HIF-1 $\alpha$ in tumors and additionally help with the identification of HIF-1 $\alpha$-targeted drugs. In addition, via the establishment of cells that demonstrate co-expression of HIF-1 $\alpha$ and GFP, the role of HIF-1 $\alpha$ in regulation of proliferation and invasion of tumor cells may be observed.

The present study constructed a lentivirus with stable HIF-1 $\alpha$ expression, and screened the stable SW480-HIF1 $\alpha$ cell line. Transwell migration assays demonstrated that following overexpression of HIF-1 $\alpha$, cell migration and proliferation were enhanced. This is in agreement with the results of previous studies that have investigated HIF-1 $\alpha$ in cancer cells (20). In the present study, following intraperitoneal injection into nude mice, the cells formed large tumor nodules, and these nodules were identified as resulting from SW480-HIF-1 $\alpha$ cells by using fluorescence microscopy. This method provides a novel in vivo model of HIF-1 $\alpha$, and in future studies, the tracking of GFP fluorescence may indicate the relevant tumor localization in vivo.

The present study method has certain limitations, for example, the process of vector construction and validation was time-consuming. The main steps included obtaining cDNA, stably-expressing HIF-1 $\alpha$ plasmid construction, lentivirus packaging and infection, stable cell line screening and tumorigenicity analysis. This multi-stage process is not conducive for large-scale laboratory applications. It is possible that diverse cDNA sequences could be obtained through collaboration and commercialization of laboratories in the future, thus reducing the amount of time required for the cloning procedure. Furthermore, during the insertion of exogenous fragments, due to the limitations of the lentiviral vector itself, the restriction sites of the enzyme multiple cloning site were relatively small, and this made it difficult to choose target sites for the primers. To facilitate clone construction, more unique rare restriction site for enzymes could be arranged, including AscI, SwaI and PmeI, and other cleavage sites. Further improvements to the efficiency of transfection would significantly shorten the screening time of stable cells by virus infection, and thus reduce the overall testing process. The present study did not use the conventional calcium phosphate transfection method, mainly as calcium phosphate transfection reagent is generally prepared by onsite laboratory personnel themselves, with a lack of appropriate quality control procedures, and also due to the different processing techniques used to prepare phosphate-plasmid complexes causing a relatively large difference in the size of the formed particles, thus an unstable transfection efficiency and unreliable results.

The small animal in vivo imaging system was a novel animal imaging system introduced in recent years, which could be used for the location of fluorescent labeled compounds, peptides, cells and nucleic acids in rats or mice. However, as small animal in vivo imaging exhibited a poor resolution for GFP, the green fluorescence produced could not be effectively detected through the skin of nude mice and there were autofluorescence interference problems at the skin surface. Therefore, in the present study, the nature of the nodules was determined after the mice were dissected, as good imaging data could not be obtained using the small animal imaging system. The techniques used in the present study are outdated compared with those used in a previous study, which reported the use of GFP to obtain in vivo imaging (21). Subsequent studies should focus on substituting luciferase for GFP in the plasmids. Following injection of the luciferase substrate into mouse models, the cold light could be motivated more easily to penetrate animal skin, and is more sensitive compared with the GFP method used in the present study (22). Future studies could be focused on investigating the downstream proteins in the HIF-1 $\alpha$ cascades and clarifying the role of these proteins in tumor proliferation, cell cycle processes and tumor invasion.

In conclusion, the lentiviral technology employed in the present study achieved the stable expression of HIF-1 $\alpha$, an important protein in the development of CRC, and a GFP tag, which allows for observation of the protein in vivo and in vitro. Lentiviral technology may provide reliable expression of proteins for the study of chronic and acute hypoxia-related diseases, such as stroke (23), high altitude pulmonary edema and obstructive sleep apnea syndrome, as well as in vivo models of disease.

\section{Acknowledgements}

The present study was funded and supported by the National Natural Science Foundation of China (grant no. 51003078) and Shanghai Science and Technology Special Funding for Laboratory Animals (grant no. 12140902302).

\section{References}

1. Siegel R, Desantis C and Jemal A: Colorectal cancer statistics, 2014. CA Cancer J Clin 64: 104-117, 2014

2. Nishimoto A, Kugimiya N, Hosoyama T, Enoki T, Li TS and Hamano K: HIF-1 $\alpha$ activation under glucose deprivation plays a central role in the acquisition of anti-apoptosis in human colon cancer cells. Int J Oncol 44: 2077-2084, 2014.

3. Cao D, Hou M, Guan YS, Jiang M, Yang Y and Gou HF: Expression of HIF-1 $\alpha$ and VEGF in colorectal cancer: Association with clinical outcomes and prognostic implications. BMC Cancer 9: 432, 2009.

4. Kim SE, Shim KN, Jung SA, Yoo K and Lee JH: The clinicopathological significance of tissue levels of hypoxia-inducible factor- $1 \alpha$ and vascular endothelial growth factor in gastric cancer. Gut Liver 3: 88-94, 2009.

5. Lucarini G, Zizzi A, Belvederesi L, Kyriakidou K, Mazzucchelli $\mathrm{R}$ and Biagini G: Increased VEGF165 expression in HCT116 colon cancer cells after transient transfection with a GFP vector encoding HIF-1 gene. J Exp Clin Cancer Res 26: $515-519,2007$. 
6. Zhong H, De Marzo AM, Laughner E, Lim M, Hilton DA Zagzag D, Buechler P, Isaacs WB, Semenza GL and Simons JW: Overexpression of hypoxia-inducible factor $1 \alpha$ in common human cancers and their metastases. Cancer Res 59: 5830-5835, 1999.

7. Hongo K, Tsuno NH, Kawai K, Sasaki K, Kaneko M, Hiyoshi M, Murono K, Tada N, Nirei T, Sunami E, et al: Hypoxia enhances colon cancer migration and invasion through promotion of epithelial-mesenchymal transition. J Surg Res 182: 75-84, 2013.

8. Dekervel J, Hompes D, van Malenstein H, Popovic D, Sagaert X, De Moor B, Van Cutsem E, D'Hoore A, Verslype C and van Pelt J: Hypoxia-driven gene expression is an independent prognostic factor in stage II and III colon cancer patients. Clin Cancer Res 20: 2159-2168, 2014

9. Ciafrè SA, Niola F, Giorda E, Farace MG and Caporossi D: $\mathrm{CoCl}(2)$-simulated hypoxia in skeletal muscle cell lines: Role of free radicals in gene up-regulation and induction of apoptosis. Free Radic Res 41: 391-401, 2007.

10. Cockrell AS and Kafri T: Gene delivery by lentivirus vectors. Mol Biotechnol 36: 184-204, 2007.

11. Ciuffi A: Mechanisms governing lentivirus integration site selection. Curr Gene Ther 8: 419-429, 2008.

12. McGarrity GJ, Hoyah G, Winemiller A, Andre K, Stein D, Blick G, Greenberg RN, Kinder C, Zolopa A, Binder-Scholl G, et al: Patient monitoring and follow-up in lentiviral clinical trials. J Gene Med 15: 78-82, 2013.

13. Kubis HP, Hanke N, Scheibe RJ and Gros G: Accumulation and nuclear import of HIF1 alpha during high and low oxygen concentration in skeletal muscle cells in primary culture. Biochim Biophys Acta 1745:187-195, 2005.

14. Zhang B, Guo W, Yu L, Wang F, Xu Y, Liu Y and Huang C: Cobalt chloride inhibits tumor formation in osteosarcoma cells through upregulation of HIF-1 $\alpha$. Oncol Lett 5: 911-916, 2013.

15. Wang V, Davis DA, Haque M, Huang LE and Yarchoan R: Differential gene up-regulation by hypoxia-inducible factor-1 alpha and hypoxia-inducible factor-2 alpha in HEK293T cells. Cancer Res 65: 3299-3306, 2005.
16. Onnis B, Rapisarda A and Melillo G: Development of HIF-1 inhibitors for cancer therapy. J Cell Mol Med 13: 2780-2786, 2009.

17. Talks KL, Turley H, Gatter KC, Maxwell PH, Pugh CW, Ratcliffe PJ and Harris AL: The expression and distribution of the hypoxia-inducible factors HIF-1alpha and HIF-2alpha in normal human tissues, cancers, and tumor-associated macrophages. Am J Pathol 157:411-421, 2000.

18. Buffa FM, West C, Byrne K, Moore JV and Nahum AE: Radiation response and cure rate of human colon adenocarcinoma spheroids of different size: The significance of hypoxia on tumor control modelling. Int J Radiat Oncol Biol Phys 49: 1109-1118, 2001

19. Ryan HE, Lo J and Johnson RS: HIF-1 alpha is required for solid tumor formation and embryonic vascularization. EMBO J 17: 3005-3015, 1998.

20. Tsai YP and Wu KJ: Hypoxia-regulated target genes implicated in tumor metastasis. J Biomed Sci 19: 102, 2012

21. Li ZH, Liao W, Cui XL, Zhao Q, Liu M, Chen YH, Liu TS Liu NL, Wang F, Yi Y, et al: Intravenous transplantation of allogeneic bone marrow mesenchymal stem cells and its directional migration to the necrotic femoral head. Int J Med Sci 8: 74-83, 2011.

22. Choy G, O'Connor S, Diehn FE, Costouros N, Alexander HR, Choyke P and Libutti SK: Comparison of noninvasive fluorescent and bioluminescent small animal optical imaging. Biotechniques 35: 1022-1026, 1028-1030, 2003.

23. Ralph GS, Parham S, Lee SR, Beard GL, Craigon MH, Ward N, White JR, Barber RD, Rayner W, Kingsman SM, et al: Identification of potential stroke targets by lentiviral vector mediated overexpression of HIF-1 alpha and HIF-2 alpha in a primary neuronal model of hypoxia. J Cereb Blood Flow Metab 24: 245-258, 2004 